\title{
Optical response and ultrafast carrier dynamics of the silicene-silver interface
}

\author{
Eugenio Cinquanta, ${ }^{1, *}$ Guido Fratesi, ${ }^{2,3, \dagger}$ Stefano dal Conte, ${ }^{4,5}$ Carlo Grazianetti, ${ }^{1}$ Francesco Scotognella, ${ }^{5}$ Salvatore Stagira, ${ }^{5}$ \\ Caterina Vozzi, ${ }^{4}$ Giovanni Onida, ${ }^{2,3}$ and Alessandro Molle ${ }^{1}$ \\ ${ }^{1}$ Laboratorio MDM, IMM-CNR, via C. Olivetti 2, Agrate Brianza, I-20864, Italy \\ ${ }^{2}$ Dipartimento di Fisica, Università degli Studi di Milano, Via Celoria 16, 20133 Milano, Italy \\ ${ }^{3}$ European Theoretical Spectroscopy Facility, Via Celoria 16, 20133 Milano, Italy \\ ${ }^{4}$ CNR Istituto di Fotonica e Nanoteconologie, Piazza Leonardo da Vinci 32, 20133 Milano, Italy \\ ${ }^{5}$ Dipartimento di Fisica, Politecnico di Milano, Piazza L. da Vinci 32, 20133 Milano, Italy \\ (Received 23 June 2015; revised manuscript received 10 September 2015; published 22 October 2015)
}

\begin{abstract}
We report a combined experimental and theoretical study of the optical response of epitaxial silicene on silver. The silicene $/ \operatorname{Ag}(111)$ ultraviolet-visible absorption spectra, which turn out to be strongly nonadditive, are analyzed in the framework of $a b$ initio calculations. Electronic transitions involving silver states are found to provide huge contributions to the optical absorption of silicene, compatible with a strong Si-Ag hybridization. The results are independent of the specific silicene configuration and are also worked out for thin amorphous silicon. This points to a dimensionality-driven peculiar dielectric response of the two-dimensional-silicon/silver interface, which is confirmed by means of transient-reflectance spectroscopy. The latter shows a metalliclike relaxation time, hence demonstrating the effects of the strong hybridization arising in silicene $/ \mathrm{Ag}(111)$ systems on charge carriers dynamical properties.
\end{abstract}

DOI: 10.1103/PhysRevB.92.165427

PACS number(s): 78.47.jg, 61.48.-c, 78.30.-j, 78.67.-n

Recently, the integration of silicene in field-effect transistors (FET) [1] opened new challenges in the comprehension of the chemical and physical properties of this elusive two-dimensional (2D) allotropic form of silicon. Intense efforts have been devoted to the study of the epitaxial silicene $/ \operatorname{Ag}(111)$ system in order to elucidate the presence of massless Dirac fermion in analogy with graphene $[2,3]$. Although recent experiments report on the linear dispersive bands [4], strong hybridization effects have been invoked as responsible for the disruption of $\pi$ and $\pi^{*}$ bands in silicene superstructures on silver [5-9]. In this framework, the measured ambipolar effect in silicene-based FET characterized by a relatively high mobility when $\mathrm{Ag}$ is withdrawn, points to a complex physics at the silicene-silver interface, demanding a deeper comprehension of its details on the atomic scale.

The present paper aims at elucidating the role of the $\operatorname{Ag}(111)$ metallic support in determining the physical properties of the $\mathrm{Si} / \mathrm{Ag}(2 \mathrm{D})$ interface, by means of optical techniques combined with theoretical calculations. In particular we show that interface states built up by mixed silicon and silver wave functions have a striking impact on the optical response of the low-dimensional $\mathrm{Si} / \mathrm{Ag}$ system.

The synthesis and the $\mathrm{Al}_{2} \mathrm{O}_{3}$ capping of our samples has been extensively described elsewhere $[1,10-12]$ and is briefly described in the Supplemental Material [13]. The silicene samples analyzed in the present paper are characterized by the simultaneous presence of the $4 \times 4$ and the $\sqrt{13} \times \sqrt{13}$ superstructures (mixed-phase silicene). We also succeed in isolating the $2 \sqrt{3} \times 2 \sqrt{3}$, as previously reported $[1,11]$.

We perform ex situ Raman characterization by using a Renishaw Invia spectrometer equipped with the $2.5 \mathrm{eV} / 488$ $\mathrm{nm}$ line of an $\mathrm{Ar}^{+}$laser line focused on the sample by a $50 \times 0.75$ N.A. Leica objective providing a spot diameter of

\footnotetext{
*eugenio.cinquanta@mdm.imm.cnr.it

†guido.fratesi@unimi.it
}

about $0.8 \mu \mathrm{m}$. The power at the sample was maintained at $1 \mathrm{~mW}$ in order to prevent laser induced sample heating, and we acquired hundreds of spectra in order to get the highest signal to noise ratio. We carried out all the measurements in a $z$-backscattering geometry.

Figure 1 compares the typical Raman spectrum of mixed-phase $(4 \times 4$ and $\sqrt{13} \times \sqrt{13})$ and $2 \sqrt{3} \times 2 \sqrt{3}$ silicene $/ \operatorname{Ag}(111)$ samples with those of a thin amorphous silicon layer (a-Si in the following) control sample (see Supplemental Material [13] for scanning tunneling microscopy images). The figure clearly shows how much the Raman spectra of the silicene/ $\operatorname{Ag}(111)$ systems differ from the one of $1 \mathrm{ML}$ equivalent a-Si. This can be easily understood in terms of the crystalline order of silicene, responsible for the narrow Raman peak, whereas the a-Si bears only short-range order resulting in a broad Raman bump peaked at $480 \mathrm{~cm}^{-1}$. The perturbation of the $\mathrm{D}_{3 d}$ point group symmetry with respect to $1 \times 1$ free standing silicene (FSS) induced by the Ag substrate triggers the activation of the breathinglike modes that in turn affect the Raman response of differently oriented phases as a function, for instance, of the exciting wavelength [11].

In this respect, the optical properties of silicene have been theoretically studied for the unsupported FSS $1 \times 1$ phase. Intriguingly, the presence of the buckling, which is responsible for the $s p^{2}-s p^{3}$ character of FSS, does not lift the degeneracy nor the linear dispersion of bands at the $K$ point in the Brillouin zone, so that FSS shows an absorbance $A(\omega) \rightarrow \pi \alpha$ (with $\alpha$ the fine-structure constant) in the limit of $\omega \rightarrow 0$. Other features are a first absorption peak at $1.6 \mathrm{eV}$ due to a van Hove singularity in the joint density of state and a main peak at about $4 \mathrm{eV}$ [14].

Concerning epitaxial silicene, the influence of the $\operatorname{Ag}(111)$ support on the band structure has been deeply studied in the literature, and it has been demonstrated that the strong $\mathrm{Si}-\mathrm{Ag}$ hybridization effect completely suppresses $\pi$ and $\pi^{*}$ bands, with the consequent loss of the Dirac cones in the silicene band structure near the $K$ points [5,7]. Nevertheless, the optical 


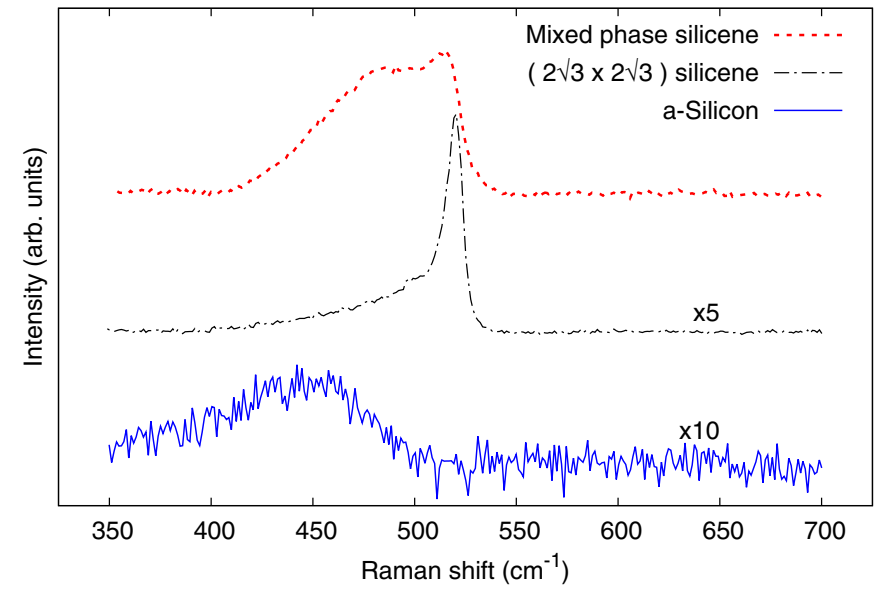

FIG. 1. (Color online) Raman spectra of mixed-phase and $2 \sqrt{3} \times 2 \sqrt{3}$ silicene/ $\operatorname{Ag}(111)$ samples (red dotted and black dashdotted curve, respectively) compared to a-Si one (blue continuous curve). The strong structure at about $520 \mathrm{~cm}^{-1}$ corresponds to the Raman-active optical modes (stretching modes) of silicene. Spectra are vertically stacked for clarity.

properties are largely unexplored in the literature; it is thus interesting to elucidate how the Ag-related loss of symmetry together with the concomitant strong $\mathrm{Si}-\mathrm{Ag}$ interaction deeply affects the dielectric response of the 2D silicene/Ag interface.

We carried out our calculations in the density-functional theory (DFT) framework. Optimized coordinates for the adsorbed silicene phases $(4 \times 4, \sqrt{13} \times \sqrt{13}$, and $2 \sqrt{3} \times 2 \sqrt{3})$ were determined by some of us in a previous paper [11] and are taken here as a reference. They are depicted in Figs. 2(a), 2(b), and 2(c), respectively. Structural models consist of a five layer $\operatorname{Ag}(111)$ slab, with silicon atoms adsorbed on one side only. Periodic boundary conditions are imposed, with a vacuum separation between adjacent slabs of at least $17 \AA$. We also considered structural models for amorphous silicon overlayers, using the same computational setup. In this case, the initial structures were obtained by slicing the atomic positions at a cubic-Si/amorphous-Si interface, as determined by tight-binding molecular dynamics simulations [15], placing it on a $4 \times 4 \operatorname{Ag}(111)$ supercell, and performing further geometrical relaxation. This resulted in the structural model shown in Fig. 2(d). The ground state charge density and potential were computed at fixed atomic coordinates by using pseudopotentials and a plane wave basis set, as implemented in the Quantum-ESPRESSO package [16]. We adopt the local density approximation (LDA) to the exchange and correlation functional [17], with a kinetic energy cutoff of 32 Ry and a $3 \times 3$ surface Brillouin zone sampling. We finally evaluate the optical properties using the Yambo software [18]. We focus on the optical absorbance at normal incidence in the visible-near UV range, which for a silicene $/ \operatorname{Ag}(111)$ slab reads [14]:

$$
A(\omega)=\frac{\omega L}{c} \operatorname{Im} \varepsilon_{M}(\omega) .
$$

Here $L$ is the thickness of the three-dimensional supercell (slab and vacuum space) in the direction orthogonal to the surface, and $\varepsilon_{M}$ its macroscopic dielectric constant. In the independent
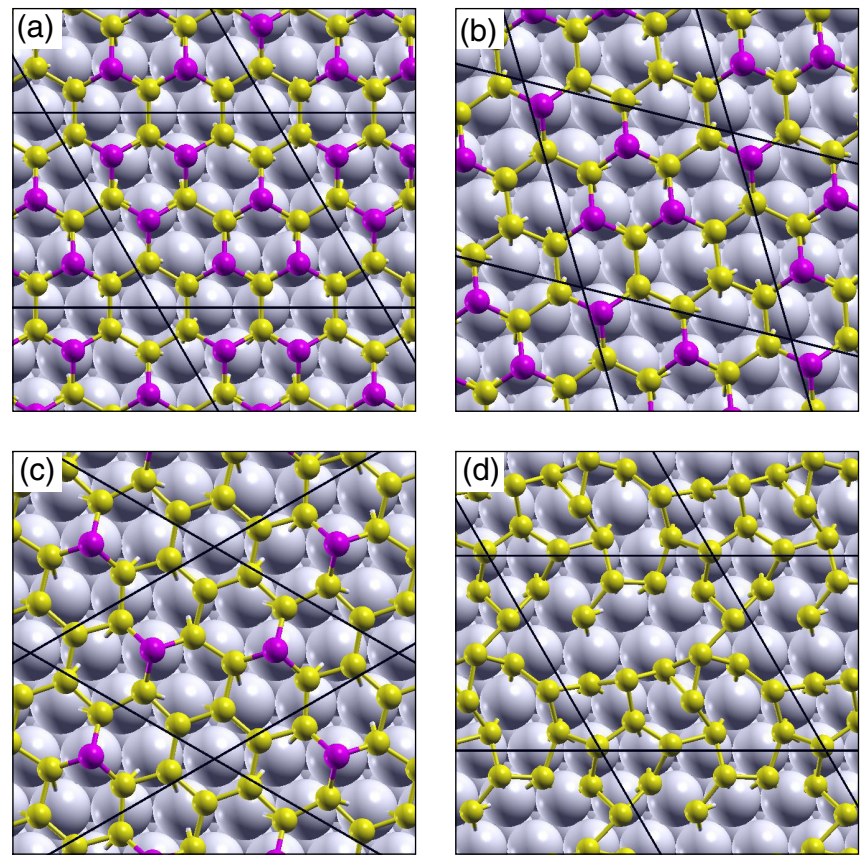

FIG. 2. (Color online) Top view of structural models for the $4 \times 4, \sqrt{13} \times \sqrt{13}$, and $2 \sqrt{3} \times 2 \sqrt{3}$ silicene $/ \operatorname{Ag}(111)$ structures are depicted in panels (a), (b), and (c). Panel (d) shows a structural model for a-Si/Ag(111). Smaller yellow and larger magenta spheres stand for planar and buckled Si atoms, respectively. The black lines mark the surface unit cells.

particle random phase approximation (IP-RPA) $\varepsilon_{M}$ is given by a sum of individual valence $(v)$ to conduction $(c)$ transitions involving the velocity operator $\mathbf{v}[19]$ :

$$
\operatorname{Im} \varepsilon_{M}(\omega)=\frac{16 \pi}{\omega^{2}} \sum_{v, c, k}\left|\left\langle\psi_{v k}|\mathbf{v}| \psi_{c k}\right\rangle\right|^{2} \delta\left(\epsilon_{c k}-\epsilon_{v k}-\omega\right) .
$$

By $v$ and $c$ we label the LDA eigenvalues $(\epsilon)$ and eigenfunctions $(\psi)$, with $k$ spanning a $12 \times 12$ mesh in the twodimensional Brillouin zone for the $4 \times 4$ case (increased to $18 \times 18$ for unsupported silicene in the same unit cell). Intraband transitions, responsible for the infrared absorbance (Drude peak) in metallic systems, were not included. It must be stressed that the inclusion of self-energy and excitonic effects has been shown to lead to a large compensation between the latter (electron-hole interaction) and the modification of the single-particle excitation energies [20]; also other silicon low-dimensional systems mostly exhibit a blueshift of the absorption peaks when going beyond IP-RPA [21].

Figure 3 shows the calculated absorption spectra of supported and unsupported $4 \times 4$ (i.e., without the $\mathrm{Ag}$ atoms but with the same $\mathrm{Si}$ coordinates) compared to that of an a-Si layer with the same density. For the unsupported $4 \times 4$ case (dotted red curve in Fig. 3), the sharp interband peak at $4 \mathrm{eV}$ characterizing FSS absorbance [14] evolves to a broader feature, still centered at $4 \mathrm{eV}$, indicating a modified band structure. Conversely, the van Hove feature at about $1.6 \mathrm{eV}$ at the $M$ point of the Brillouin zone for FSS is still present, as shown in the inset of Fig. 3, indicating that the singularity in the band structure at this energy is preserved despite the change in lattice structure and point-group symmetry $\left(\mathbf{C}_{6 v}\right)$. However, 


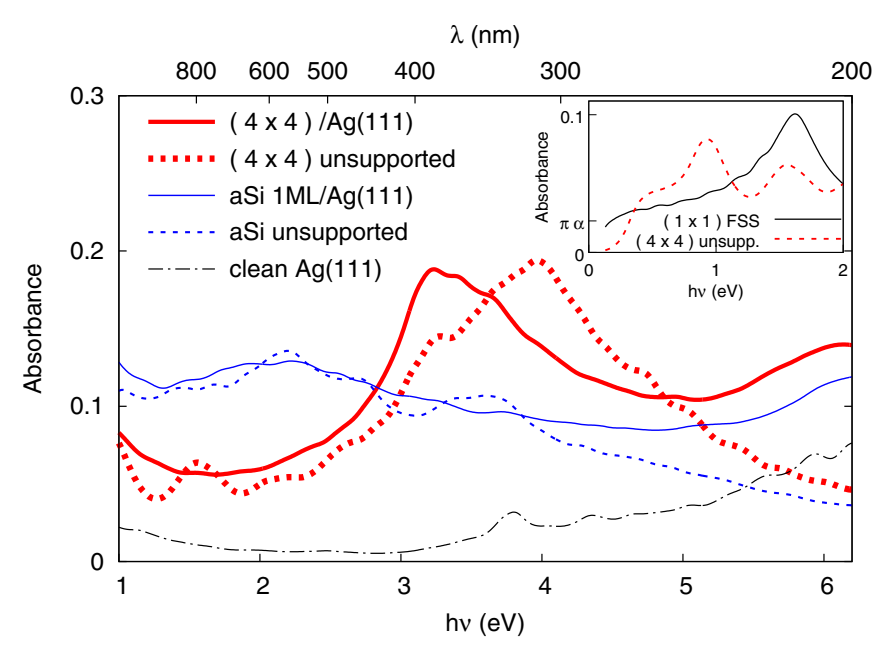

FIG. 3. (Color online) Calculated absorbance of the Agsupported and unsupported $4 \times 4$ superstructure compared to the supported and unsupported amorphous silicon. The absorbance of the bare Ag slab is also reported. The inset compares the absorbances of $1 \times 1$ FSS and unsupported $4 \times 4$ in the infrared range.

that symmetry breaking of the $4 \times 4$ atomic arrangement opens a band gap at $\Gamma$ of $0.30 \mathrm{eV}$, in agreement with previous findings [5,22], so that $A(\omega)=0$ at lower frequencies, as can be seen in the inset of Fig. 3, which compares the $1 \times 1$ FSS and unsupported $4 \times 4$ absorbances in the infrared range. If the crystalline order is totally reduced down to the amorphous limit, as for the unsupported a-Si (blue dotted line in Fig. 3), then all the absorption peaks disappear and the absorbance is almost flat all over the UV-Vis spectral region with a slight maximum at $2 \mathrm{eV}$.

The effect of the silver substrate on the optical response of the Ag supported silicene is not trivial and reflects the special physics of the 2D silicene/Ag interface. The dash-dotted curve in Fig. 3 reports the absorption of the bare silver slab, which puts into evidence a negligible contribution in the visible spectral range and a significant absorbance above $4 \mathrm{eV}$. We neglect here the Drude tail, which cannot be accounted for by the present treatment and will not be discussed further. The continuous red curve in Fig. 3 is the absorbance of $4 \times 4$ silicene on $\mathrm{Ag}(111)$. Clearly, the presence of the substrate does not trivially recast as a modified background, given by the sum between the absorbance of the bare $\mathrm{Ag}(111)$ slab and of the unsupported silicene. Beyond an increased background in the UV spectral region, two main effects are evident: the absence of the van Hove peak at $1.6 \mathrm{eV}$, combined with the sharpening and the redshifting of the main absorption peak to about $3.3 \mathrm{eV}$. The former fact highlights how the presence of silver deeply impacts the band structure of the adsorbed silicon, and consequently its absorbance: This finding is consistent with a strong hybridization between $\mathrm{Ag}$ and $\mathrm{Si}$ states, as previously argued [5,7-9]. These effects are apparently less evident for the a-Si case, whose absorption profile (continuous blue line in Fig. 3) is relatively less structured, and the major influence of the underlying silver substrate is a further smoothening of spectral features (dashed blue line).

In order to elucidate the role of silver in determining the optical response of epitaxial silicene, we developed a method enabling us to factor the contribution to the optical absorption due to valence and conduction states localized either on $\mathrm{Si}$ or Ag atoms. This can be evaluated from the projected amplitude of the Kohn-Sham eigenstate $\psi_{i k}$ on the atomic orbitals $\phi$ of the given species $X(X=\mathrm{Si}, \mathrm{Ag})$, defined as standard: $W_{i k}^{X}=\sum_{\phi \in X}\left|\left\langle\phi \mid \psi_{i k}\right\rangle\right|^{2}$. The contribution to the absorbance from valence $v$ and conduction $c$ states localized on species $X$ and $Y$, respectively, is then computed by modifying Eq. (2) as follows:

$$
\begin{aligned}
\operatorname{Im} \varepsilon_{M}^{X Y}(\omega)= & \frac{16 \pi}{\omega^{2}} \sum_{v, c, k} W_{v k}^{X} W_{c k}^{Y}\left|\left\langle\psi_{v k}|\mathbf{v}| \psi_{c k}\right\rangle\right|^{2} \\
& \times \delta\left(\epsilon_{c k}-\epsilon_{v k}-\omega\right) .
\end{aligned}
$$

Hence the total absorbance is given by the sum of pure terms $(\mathrm{Si} \rightarrow \mathrm{Si}, \mathrm{Ag} \rightarrow \mathrm{Ag})$ and mixed ones $(\mathrm{Si} \rightarrow \mathrm{Ag}, \mathrm{Ag} \rightarrow \mathrm{Si})$. Since the projection is not complete, one also has a remainder due to orbitals other than $\mathrm{Si}$ and $\mathrm{Ag}$ ones, which in our case constitutes a minimal fraction of the spectrum.

Figures 4(a), 4(b), and 4(c) show the optical absorption of the three considered supported silicene phases factorized in pure and mixed contributions [23]. Remarkably, also in the spectral region where the absorption is strictly related to the presence of silicene (about 3-4 eV, as shown in Fig. 3), the pure silicon states provide just a weak contribution to the total absorption whereas electronic transitions involving pure $\mathrm{Ag}$ states or mixed $\mathrm{Si} \rightarrow \mathrm{Ag}$ and $\mathrm{Ag} \rightarrow \mathrm{Si}$ states are responsible for almost the whole intensity. This apparently counterintuitive result puts into evidence delocalization and hybridization effects involving the electronic states at the $\mathrm{Si} / \mathrm{Ag}$ interface, irrespectively of the local atomic arrangement in the epitaxial silicene lattice: On the one hand a distinction between spectral features attributed to the presence of silicene on $\operatorname{Ag}(111)$ can be clearly identified, despite the modifications with respect to FSS discussed above, so that electronic states at the interface bear the signature of the Si overlayer in the spectrum. On the other hand, the strong interaction between the adsorbate and the metallic support delocalizes silicon-induced states well beyond the silicon region itself.

Figure 4(d) shows the factorized absorption of $1 \mathrm{ML}$ of a-Si on $\mathrm{Ag}(111)$. We recall that for this system the presence of the substrate has a much weaker influence on the absorption of the unsupported layer (see Fig. 3) than for $4 \times 4$ silicene. Nevertheless, also for a-Si we recover the same hybridization effects observed for silicene. While the absence of modification in the visible spectrum of supported and unsupported a-Si is straightforward, the contribution of $\mathrm{Ag}$ wave functions to the absorption observed in Fig. 4(d) is remarkable. In complete analogy with the silicene case, a-Si optical absorption is dominated by pure $\mathrm{Ag}$ and mixed $\mathrm{Ag} \rightarrow \mathrm{Si} / \mathrm{Si} \rightarrow \mathrm{Ag}$ contributions, providing evidence that the hybridization of $\mathrm{Si}$ wave functions with those of the silver substrate is independent of the atomic configuration of the $\mathrm{Si}$ layer. This remarkable effect thus emerges as strictly related to the $2 \mathrm{D}$ nature of the silicon adsorbate and its interaction with the substrate.

In order to further investigate this point, we performed transient-reflectance optical spectroscopy, a time-resolved experimental technique giving access to the study of the carrier dynamics on the electronic timescale. We hence investigate the ultrafast photophysical properties of silicene by performing 


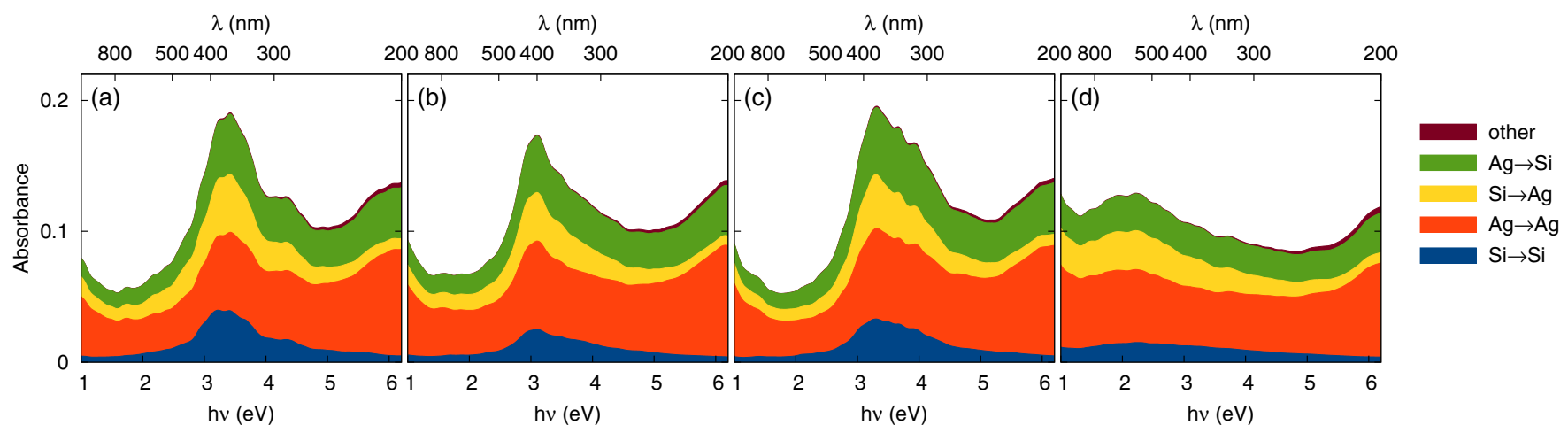

FIG. 4. (Color online) Factorized absorbance of (a) $4 \times 4$, (b) $\sqrt{13} \times \sqrt{13}$, and (c) $2 \sqrt{3} \times \sqrt{3}$ silicene/Ag(111) phases, and (d) a-Si into the various valence $\rightarrow$ conduction contributions, defined according to Eq. (3).

pump-probe measurements in reflection geometry, with a temporal resolution of about $20 \mathrm{fs}$. We excited the sample with a broadband visible pulse, spectrally peaked around $500 \mathrm{~nm}$, obtained from a visible optical parametric amplifier (OPA) [24]. We used as a probe the second harmonic of another OPA. Both OPAs were driven by an amplified Ti:sapphire laser system $(500 \mu \mathrm{J}, 150 \mathrm{fs}, 1 \mathrm{kHz})$. After chirped mirror compression, the duration of the pump pulse was less than 15 fs. We measured the probe reflection of the sample with an optical multichannel analyzer working at the full repetition rate of the laser source. The acquisition of the pump-perturbed and pump-unperturbed probe spectra allowed us to extract the sample differential reflectivity $\frac{\Delta R}{R}$ in mixedphase silicene/ $\operatorname{Ag}(111), 2 \sqrt{3} \times 2 \sqrt{3}$ silicene/ $\operatorname{Ag}(111)$, and a-Si $/ \mathrm{Ag}(111)$. Since all the systems show a strong absorption at $340 \mathrm{~nm}$ (3.65 eV, see Supplemental Material for 2D pump and probe maps [13]), we show in Fig. 5 the transient reflectivity change as a function of pump-probe delay for the probe wavelength of $340 \mathrm{~nm}$ in mixed-phase silicene/Ag(111) (red dotted curve), $2 \sqrt{3} \times 2 \sqrt{3}$ silicene/Ag(111) (black dashdotted curve), and a-Si/Ag(111) (blue continuous curve). The absorption at $340 \mathrm{~nm}$ is characterized by an ultrafast

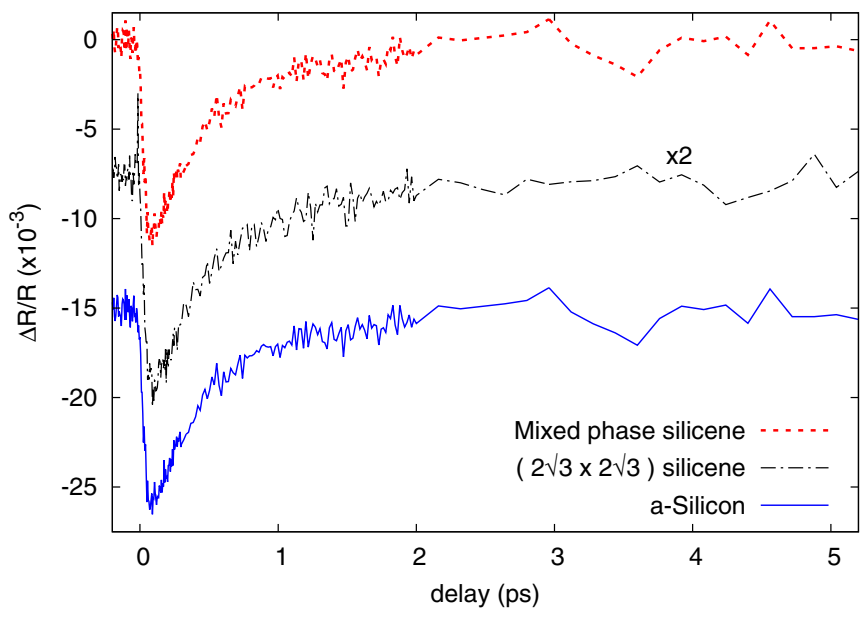

FIG. 5. (Color online) Transient-reflectance spectra as a function of pump-probe delay for the probe wavelength of $340 \mathrm{~nm}$ in mixed-phase silicene/ $\operatorname{Ag}(111)$ (red dotted curve), $2 \sqrt{3} \times 2 \sqrt{3}$ silicene/ $\operatorname{Ag}(111)$ (black dash-dotted curve), and a-Si/Ag(111) (blue continuous curve). Spectra are vertically stacked for clarity. decay in the first 2 ps after the excitation. Bulk diamondlike and amorphous silicon typically show charge dynamics with relaxation time of the order of tens/hundreds of ps due to long recombination time of excitons in indirect and direct band-gap semiconductors [25,26]. On the other hand, metals are characterized by ultrafast dynamics with a time constant of the order of tens of fs arising from e-e thermalization [27]. The carrier dynamics at the silicene/Ag interface can be inferred both for Ag-supported silicene and a-Si. In detail, carrier relaxation takes place with a time constant of hundreds of fs, reflecting a metalliclike behavior rather than a semiconducting one. Intriguingly, the reduced dimensionality of the Si layer and its coupling to the substrate induces relaxation time for the carriers two or three orders of magnitude faster than in conventional semiconductors but not as fast as in a purely metallic system (the dynamics of diamondlike silicon and the bare Ag substrate are compared in Ref. [28]), therein claiming a peculiar dynamics for both $2 \mathrm{D}$ Si adsorbates. The observed dynamics have to be ascribed to the presence of a fast relaxation path characterizing the $2 \mathrm{D} \mathrm{Si} / \mathrm{Ag}$ interface, such as e-e thermalization and e-ph scattering processes, and the absence of long e-h recombination typical for semiconductors. The delocalization effect described in Fig. 4, and the related contribution to the absorption of electronic transition involving Ag wave functions, suggests that the strong hybridization present at the $2 \mathrm{D} \mathrm{Si} / \mathrm{Ag}$ interface influences the carrier dynamics by inducing fast metalliclike relaxation channels. Nonetheless, Tao et al. [1] demonstrated that a-Si and epitaxial silicene show strikingly different electrical responses when integrated in FETs once the underlying silver support is removed, the former being trivially semiconducting and the latter exhibiting an ambipolar transport behavior. This fact provides evidence of the dual role of the substrate, which on one hand allows for the synthesis of a metastable phase of silicene and on the other hand induces a metallic character through a proximity hybridization.

In conclusion, we studied experimentally and theoretically the optical response of the $2 \mathrm{D}$ silicene/Ag interface, the paradigmatic system for the synthesis of the epitaxial silicene. We show how the metallic substrate contributes to the absorption not in a simple additive way, and we explain this effect in terms of hybridization of silicon states with silver ones extending in the metallic support. Thanks to the silver contribution the charge carrier dynamics occurs 
on timescales much faster than for typical semiconductors, yet not as fast as on pure Ag. The similarity of outcomes we find for a silver-supported ultrathin amorphous silicon layer calls for a mechanism driven by the dimensionality reduction of $\mathrm{Si}$ at the interface. Our findings can have a strong impact in the case of other supported elemental 2D materials, such as germanene [29] and stanene [30,31] deposited on commensurate substrates.

\section{ACKNOWLEDGMENTS}

The present research activity has been carried on within the 7th Framework Programme of the European Commission,
FET-OPEN call, Grant No. 270749, “2D-NANOLATTICES” project. The research leading to these results has also received funding from the ERC Starting Research Grant UDYNI (Grant No. 307964, EC Seventh Framework Programme) and from the Italian Ministry of Research and Education (ELI projectESFRI Roadmap). G.F. and G.O. thank Davide Sangalli for useful discussions. We acknowledge the CINECA Award No. HP10CESYLM, 2014 for the availability of high performance computing resources and support. S.D.C. received financial support from Futuro through Ricerca Grant No. RBFR12SW0J of the Italian Ministry of Education, University and Research. E.C., C.G., and A.M. also acknowledge funding from CNR grant "Joint Lab".
[1] L. Tao, E. Cinquanta, D. Chiappe, C. Grazianetti, M. Fanciulli, M. Dubey, A. Molle, and D. Akinwande, Nat. Nanotech. 10, 227 (2015).

[2] P. Vogt, P. De Padova, C. Quaresima, J. Avila, E. Frantzeskakis, M. C. Asensio, A. Resta, B. Ealet, and G. Le Lay, Phys. Rev. Lett. 108, 155501 (2012).

[3] L. Chen, H. Li, B. Feng, Z. Ding, J. Qiu, P. Cheng, K. Wu, and S. Meng, Phys. Rev. Lett. 110, 085504 (2013).

[4] Y. Feng, D. Liu, B. Feng, X. Liu, L. Zhao, Z. Xie, Y. Liu, A. Liang, C. Hu, Y. Hu, S. He, G. Liu, J. Zhang, C. Chen, Z. Xu, L. Chen, K. Wu, Y.-T. Liu, H. Lin, Z.-Q. Huang, C.-H. Hsu, F.-C. Chuang, A. Bansil, and X. J. Zhou, arXiv:1503.06278.

[5] S. Cahangirov, M. Audiffred, P. Tang, A. Iacomino, W. Duan, G. Merino, and A. Rubio, Phys. Rev. B 88, 035432 (2013).

[6] C.-L. Lin, R. Arafune, K. Kawahara, M. Kanno, N. Tsukahara, E. Minamitani, Y. Kim, M. Kawai, and N. Takagi, Phys. Rev. Lett. 110, 076801 (2013).

[7] D. Tsoutsou, E. Xenogiannopoulou, E. Golias, P. Tsipas, and A. Dimoulas, Appl. Phys. Lett. 103, 231604 (2013).

[8] S. K. Mahatha, P. Moras, V. Bellini, P. M. Sheverdyaeva, C. Struzzi, L. Petaccia, and C. Carbone, Phys. Rev. B 89, 201416 (2014).

[9] H. Ishida, Y. Hamamoto, Y. Morikawa, E. Minamitani, R. Arafune, and N. Takagi, New J. Phys. 17, 015013 (2015).

[10] D. Chiappe, C. Grazianetti, G. Tallarida, M. Fanciulli, and A. Molle, Adv. Mater. 24, 5088 (2012).

[11] E. Cinquanta, E. Scalise, D. Chiappe, C. Grazianetti, B. van den Broek, M. Houssa, M. Fanciulli, and A. Molle, J. Phys. Chem. C 117, 16719 (2013).

[12] A. Molle, C. Grazianetti, D. Chiappe, E. Cinquanta, E. Cianci, G. Tallarida, and M. Fanciulli, Adv. Funct. Mater. 23, 4340 (2013)

[13] See Supplemental Material at http://link.aps.org/supplemental/ 10.1103/PhysRevB.92.165427 for the description of the silicene deposition methods and 2D pump and probe maps.

[14] F. Bechstedt, L. Matthes, P. Gori, and O. Pulci, Appl. Phys. Lett. 100, 261906 (2012).

[15] I. Santos, M. Cazzaniga, G. Onida, and L. Colombo, J. Phys.: Condens. Matter 26, 095001 (2014).
[16] P. Giannozzi, S. Baroni, N. Bonini, M. Calandra, R. Car, C. Cavazzoni, D. Ceresoli, G. L. Chiarotti, M. Cococcioni, I. Dabo, A. Dal Corso, S. Fabris, G. Fratesi, S. de Gironcoli, R. Gebauer, U. Gerstmann, C. Gougoussis, A. Kokalj, M. Lazzeri, L. Martin-Samos, N. Marzari, F. Mauri, R. Mazzarello, S. Paolini, A. Pasquarello, L. Paulatto, C. Sbraccia, S. Scandolo, G. Sclauzero, A. P. Seitsonen, A. Smogunov, P. Umari, and R. M. Wentzcovitch, J. Phys.: Condens. Matter 21, 395502 (2009) (see also http://www.quantum-espresso.org).

[17] J. P. Perdew and Alex Zunger, Phys. Rev. B 23, 5048 (1981).

[18] A. Marini, C. Hogan, M. Grüning, and D. Varsano, Comp. Phys. Comm. 180, 1392 (2009).

[19] G. Onida, L. Reining, and A. Rubio, Rev. Mod. Phys. 74, 601 (2002).

[20] W. Wei and Timo Jacob, Phys. Rev. B 88, 045203 (2013).

[21] S. Cahangirov, V. O. Özçelik, Angel Rubio, and Salim Ciraci, Phys. Rev. B 90, 085426 (2014).

[22] The structure named $4 \times 4$ is labeled according to the corresponding reconstruction of the $\operatorname{Ag}(111)$ lattice, following the conventional use; indeed it corresponds to a $3 \times 3$ reconstruction of the honeycomb silicene lattice. As a consequence, the $K$ point of $1 \times 1$ FSS folds into $\Gamma$ of such a superstructure, where the gap opens [6].

[23] These results were worked out using a coarser $6 \times 6 k$-point mesh.

[24] M. Beutler, M. Ghotbi, F. Noack, D. Brida, C. Manzoni, and G. Cerullo, Opt. Lett. 34, 710 (2009).

[25] F. E. Doany and D. Grischkowsky, Appl. Phys. Lett. 52, 36 (1988).

[26] I. A. Shkrob and R. A. Crowell, Phys. Rev. B 57, 12207 (1998).

[27] N. Del Fatti, C. Voisin, M. Achermann, S. Tzortzakis, D. Christofilos, and F. Vallee, Phys. Rev. B 61, 16956 (2000).

[28] E. Cinquanta, S. D. Conte, D. Chiappe, C. Grazianetti, M. Fanciulli, A. Molle, G. Cerullo, S. Stagira, F. Scotognella, and C. Vozzi, Springer Proceedings in Physics 162, 329 (2015).

[29] M. Derivaz, D. Dentel, R. Stephan, M.-C. Hanf, A. Mehdaoui, P. Sonnet, and C. Pirri, Nano Lett. 15, 2510 (2015).

[30] Y. Xu, B. Yan, H.-J. Zhang, J. Wang, G. Xu, P. Tang, W. Duan, and S.-C. Zhang, Phys. Rev. Lett. 111, 136804 (2013).

[31] F.-f. Zhu, W.-j. Chen, Y. Xu, C.-1. Gao, D.-d. Guan, C.-h. Liu, D. Qian, S.-C. Zhang, and J.-f. Jia, Nat. Mater. 14, 1020 (2015). 